\title{
REPRESENTATION OF FUNCTIONS AS WALSH SERIES TO DIFFERENT BASES AND AN APPLICATION TO THE NUMERICAL INTEGRATION OF HIGH-DIMENSIONAL WALSH SERIES
}

\author{
GERHARD LARCHER, WOLFGANG CH. SCHMID, AND REINHARD WOLF
}

\begin{abstract}
We will prove the following theorem on Walsh series, and we will derive from this theorem an effective and constructive method for the numerical integration of Walsh series by number-theoretic methods. Further, concrete computer calculations are given.
\end{abstract}

Theorem. For base $b \geq 2$, dimension $s \geq 1$, and $\alpha>1, c>0 \quad(b, s \in$ $\mathbb{N} ; c, \alpha \in \mathbb{R})$, let ${ }_{b} \bar{E}_{s}^{\alpha}(c)$ be the class of all functions $f:[0,1)^{s} \rightarrow \mathbb{C}$ which are representable by absolutely convergent Walsh series to base $b$ with Walsh coefficients $\widehat{W}\left(h_{1}, \ldots, h_{s}\right)$ with the following property: $\left|\widehat{W}\left(h_{1}, \ldots, h_{s}\right)\right| \leq$ $c \cdot\left(\bar{h}_{1} \cdots \bar{h}_{s}\right)^{-\alpha}$ for all $h_{1}, \ldots, h_{s}$, where $\bar{h}:=\max (1,|h|)$. We show that if $f \in{ }_{2} \bar{E}_{s}^{\alpha}(c)$, then $f \in{ }_{2^{h}} \bar{E}_{s}^{\alpha-\beta_{h}}\left(c \cdot 2^{h s \alpha}\right)$ for all $h \geq 2$, provided that $\alpha>1+\beta_{h}$, where

$$
\beta_{h}=\frac{h-1}{2 h}+\frac{\sum_{k=0}^{h-2} \log \sin \left(\frac{\pi}{4}+\frac{\pi}{2}\left\{\frac{4^{\left[\frac{k}{2}\right]}-1}{3 \cdot 2^{k+1}}\right\}\right)}{h \cdot \log 2} .
$$

The "exponent" $\alpha-\beta_{h}$ is best possible for all $h$, and $\beta_{h}$ is monotonically increasing with

$$
\beta:=\lim _{h \rightarrow \infty} \beta_{h}=\frac{1}{2}+\frac{\log \sin \frac{5 \pi}{12}}{\log 2}=0.4499 \ldots
$$

\section{INTRODUCTION}

In recent times, especially in the context of image processing and signal processing, it has become necessary to give good effective methods for the numerical integration of functions which are representable by Walsh series. A first number-theoretic approach to this problem, in some (implicit) sense, was already given by Sobol $[8,9]$. (He indeed was dealing with Haar functions.) But a first systematic investigation of number-theoretic methods for the numerical integration of Walsh series with effective results and, in some sense, best possible error estimates-an investigation which only was possible on the basis of the theory of $(t, m, s)$-nets developed by Niederreiter-was worked out only quite recently in [3]. (See also [2].) We now give the basic concepts and the basic results of this theory.

Received by the editor March 8, 1993 and, in revised form, August 20, 1993.

1991 Mathematics Subject Classification. Primary 42C10, 11K45. 
Definition 1. Let $b \geq 2$ be an integer and $w:=e^{2 \pi i / b}$. The Rademacher functions to base $b$ are defined by

$$
\phi_{0}^{(b)}(x)=w^{k} \text { for } \frac{k}{b} \leq x<\frac{k+1}{b}, k=0, \ldots, b-1,
$$

and for $n \geq 0$ by

$$
\phi_{n}^{(b)}(x+1):=\phi_{n}^{(b)}(x):=\phi_{0}^{(b)}\left(b^{n} x\right) .
$$

Definition 2. The Walsh functions to base $b$ are defined by

$$
\operatorname{wal}_{0}^{(b)}(x)=1
$$

and, if $n=a_{1} b^{n_{1}}+\cdots+a_{m} b^{n_{m}}$ with $0 \leq a_{i}<b$ and $n_{1}>n_{2}>\cdots$, by

$$
\operatorname{wal}_{n}^{(b)}(x)=\phi_{n_{1}}^{a_{1}}(x) \cdots \phi_{n_{m}}^{a_{m}}(x),
$$

where $\phi$ means Rademacher to base $b$. For dimension $s \geq 2$ and $k_{1}, \ldots, k_{s} \geq$ 0 , we define

$$
\operatorname{wal}_{k_{1}, \ldots, k_{s}}^{(b)}\left(x_{1}, \ldots, x_{s}\right)=\operatorname{wal}_{k_{1}}^{(b)}\left(x_{1}\right) \cdots \operatorname{wal}_{k_{s}}^{(b)}\left(x_{s}\right) .
$$

The set $W=\left\{\operatorname{wal}_{k_{1}, \ldots, k_{s}}^{(b)}\left(x_{1}, \ldots, x_{s}\right) \mid k_{i} \geq 0, i=1, \ldots, s\right\}$ is an orthonormal and complete system of functions in $L^{2}\left([0,1)^{s}\right)$.

Definition 3. For base $b \geq 2$, dimension $s \geq 1$, and $\alpha>1, c>0 \quad(b, s \in$ $\mathbb{N} ; c, \alpha \in \mathbb{R})$, let ${ }_{b} \bar{E}_{s}^{\alpha}(c)$ be the class of all functions $f:[0,1)^{s} \rightarrow \mathbb{C}$ which are representable by absolutely convergent Walsh series

$$
\sum_{h_{1}, \ldots, h_{s}=0}^{\infty} \widehat{W}\left(h_{1}, \ldots, h_{s}\right) \cdot \mathrm{wal}_{h_{1}, \ldots, h_{s}}^{(b)}\left(x_{1}, \ldots, x_{s}\right)
$$

with Walsh coefficients $\widehat{W}\left(h_{1}, \ldots, h_{s}\right)$ having the following property:

$$
\left|\widehat{W}\left(h_{1}, \ldots, h_{s}\right)\right| \leq \frac{c}{\left(\bar{h}_{1} \cdots \bar{h}_{s}\right)^{\alpha}} \text { for all }\left(h_{1}, \ldots, h_{s}\right) \neq(0, \ldots, 0) .
$$

(Here, $\bar{h}:=\max (1,|h|)$.)

Then, for given $N$, we are asking for point sets $\underline{x}_{1}, \ldots, \underline{x}_{N}$ in $[0,1)^{s}$ such that the integration error

$$
R_{N}(f):=\left|\int_{[0,1)^{s}} f(\underline{x}) d \underline{x}-\frac{1}{N} \sum_{k=1}^{N} f\left(\underline{x}_{k}\right)\right|
$$

is small for all $f \in{ }_{b} \bar{E}_{s}^{\alpha}(c)$.

It turns out that point sets achieving this objective in a best possible way are provided by so-called $(t, m, s)$-nets to base $b$, and especially by digital $(t, m, s)$-nets to base $b$, which can be defined by the following chain of definitions.

Definition 4. For an integer $b \geq 2$, an elementary interval to base $b$ is defined to be an interval of the form

$$
\prod_{i=1}^{s}\left[\frac{a^{(i)}}{b^{d_{i}}}, \frac{a^{(i)}+1}{b^{d_{i}}}\right)
$$


with integers $d_{i}, a^{(i)} \geq 0$ and $a^{(i)}<b^{d_{i}}$ for $1 \leq i \leq s$.

Definition 5. Let $b \geq 2$ and $0 \leq t \leq m$ be integers. A $(t, m, s)$-net to base $b$ is a set of $b^{m}$ points in $[0,1)^{s}$ for which we have that in every elementary interval to base $b$ of volume $b^{t-m}$ there are exactly $b^{t}$ points of the set.

Such $(t, m, s)$-nets have excellent distribution properties if $t$ is small (the optimal case of course is $t=0$ ), as was pointed out in detail in [6].

One possibility of constructing such nets is the so-called digital method. That is:

Definition 6. Let $b \geq 2$ be a given base. Let $R:=\{\overline{0}, \ldots, \overline{b-1}\}$ be an arbitrary ring with zero element $\overline{0}$. Let $C_{i}=\left(c_{k l}^{(i)}\right), k, l=1, \ldots, m, i=$ $1, \ldots, s$, be $m \times m$ matrices over $R$. In the following we identify every integer $n, 0 \leq n \leq b^{m}-1, n=a_{m-1} b^{m-1}+\cdots+a_{0}, a_{i} \in\{0, \ldots, b-1\}$, with the vector $\vec{n}:=\left(\bar{a}_{m-1}, \ldots, \bar{a}_{0}\right)^{T}$, and conversely. Then let

$$
\underline{x}_{n}:=\left(\frac{1}{b^{m}}\left(C_{1} \cdot \vec{n}\right), \ldots, \frac{1}{b^{m}}\left(C_{s} \cdot \vec{n}\right)\right) \in[0,1)^{s} .
$$

If this point set is a $(t, m, s)$-net to base $b$, then it will be called a digital $(t, m, s)$-net.

Niederreiter [6] gives the following explicit examples of digital $(0, m, s)$-nets to any prime-power base $b$, provided that $s \leq b+1$.

Construction method. Let $F_{b}$ be the Galois field of order $b$. Then we define the matrices $C_{i}=\left(c_{j r}^{(i)}\right)$ over $F_{b}, j=1, \ldots, m, r=0, \ldots, m-1, i=$ $1, \ldots, s$, in the following way: Choose $s-1$ pairwise different elements $\beta_{i}$, $i=1, \ldots, s-1$, from $F_{b}$. Let

$$
\begin{aligned}
& c_{j r}^{(i)}=0 \text { for } 1 \leq i \leq s-1,2 \leq j \leq m, 0 \leq r<j-1, \\
& c_{j r}^{(i)}=\left(\begin{array}{c}
r \\
j-1
\end{array}\right) \cdot \beta_{i}^{r-j+1} \text { for } 1 \leq i \leq s-1,1 \leq j \leq m, j-1 \leq r \leq m-1, \\
& c_{j r}^{(s)}=\delta_{r, m-j} \text { for } 1 \leq j \leq m, 0 \leq r \leq m-1 .
\end{aligned}
$$

Here, $\beta^{0}=1$ for all $\beta \in F_{b}$, and $\delta$ is the Kronecker symbol. The point set corresponding to these matrices then always is a $(0, m, s)$-net to base $b$. Two easy examples:

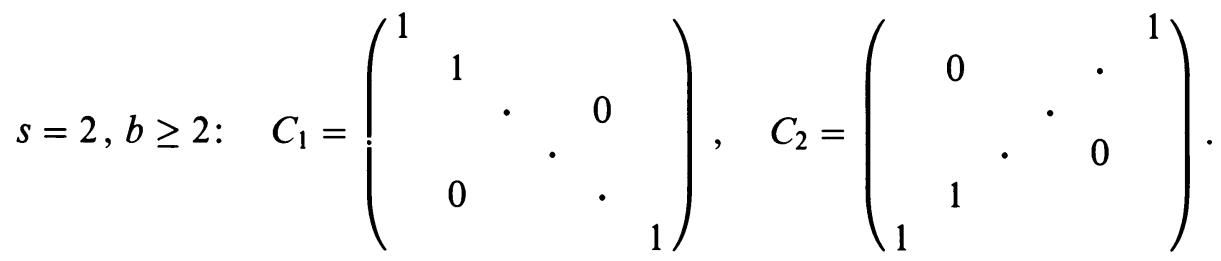

This choice of the $C_{i}$ provides the so-called Hammersley sequence to base $b$, which is well known in uniform distribution;

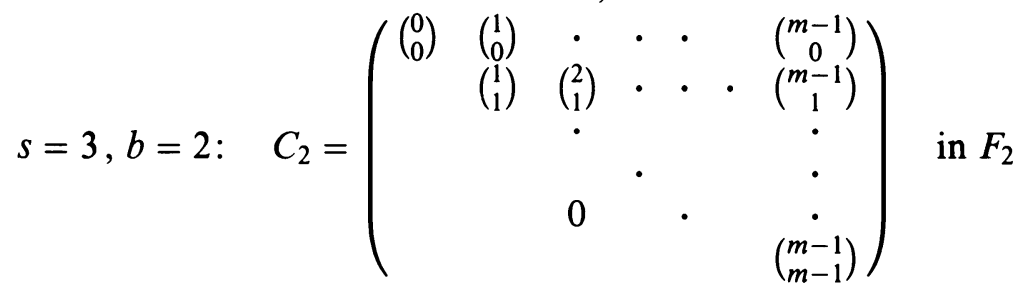


with $C_{1}$ as above and $C_{3}$ equal to the second matrix from the first example gives a $(0, m, 3)$-net to base 2 .

If we use $(t, m, s)$-nets, and especially digital $(t, m, s)$-nets, for the integration of Walsh series, then we get the following result:

Theorem 1. (a) If $\underline{x}_{1}, \ldots, \underline{x}_{N}$ is a $(t, m, s)$-net to base $b$, then, with a positive constant $K(s, \alpha, c)$ depending only on $s, \alpha$, and $c$, we have

$$
R_{N}(f) \leq K(s, \alpha, c) \cdot b^{t(\alpha-1)} \cdot \frac{(\log N)^{s-1}}{N^{\alpha-1}}
$$

for all $f \in{ }_{b} \bar{E}_{s}^{\alpha}(c)$.

(b) If $b$ is a prime, $R$ the Galois field $F_{b}$, and $\underline{x}_{1}, \ldots, \underline{x}_{N}$ a digital $(t, m, s)$-net to base $b$ defined over $R$, then, with a positive constant $K(s, \alpha, c)$ depending only on $s, \alpha$, and $c$, we have

$$
R_{N}(f) \leq K(s, \alpha, c) \cdot b^{t \alpha} \cdot \frac{(\log N)^{s-1}}{N^{\alpha}}
$$

for all $f \in{ }_{b} \bar{E}_{s}^{\alpha}(c)$.

For the proof of Theorem 1, see [3]. An attempt to prove an analog to part (b) of the theorem for prime-power case $b$ causes technical problems, so that until now, this result is proven for $b$ prime only. However, numerical investigations (see [7]) lead us to conjecture that this error estimate also holds for prime-power base.

Part (b) of Theorem 1 is in some sense the best possible result that can be obtained in any case: For the optimal case of digital nets with $t=0$, we have

$$
R_{N}(f) \leq K(s, \alpha, c) \cdot \frac{(\log N)^{s-1}}{N^{\alpha}} .
$$

On the other hand, we have the following result:

Theorem 2. For all $b \geq 2, c>0, \alpha>1, s \in \mathbb{N}$ there is a constant $c^{\prime}:=$ $c^{\prime}(c, \alpha, s, b)>0$ such that: For all $N$ and every point set $\underline{x}_{1}, \ldots, \underline{x}_{N}$ in $[0,1)^{s}$ there is an $f \in{ }_{b} \bar{E}_{s}^{\alpha}(c)$ with $f\left(\underline{x}_{k}\right)=0$ for $k=1, \ldots, N$ and

$$
\int_{[0,1)^{s}} f(\underline{x}) d \underline{x}>c^{\prime} \cdot \frac{(\log N)^{s-1}}{N^{\alpha}} \text {. }
$$

For the proof of Theorem 2, again see [3].

So to make these theoretical results effective and applicable (and this is one aim of this paper), we have to ask: For given $s, b$, and $m$, how can we find digital $(t, m, s)$-nets to base $b$ with $t$ as small as possible, and how small can $t$ be for given $s, b$, and $m$ ?

For most applications, the most important case is the case of base $b=2$. Therefore, in the following we will concentrate on practical aspects for this case. As we have seen above, we have a concrete construction method for digital $(0, m, s)$-nets to base 2 for dimension $s=2$ or 3 . For dimension $s \geq 4$, however, it was shown by Niederreiter [6] that there cannot exist a $(0, m, s)$-net to base 2 .

It need not be emphasized that methods for numerical integration are of increasing interest the higher the dimension is they work for. That is: We 
would need effective construction methods for $(t, m, s)$-nets to base 2 with small $t$ for dimensions $s$ at least 8 or 10 .

A special construction method for $(t, m, s)$-nets with $t=O(s \log s)$ for base 2 , independently of $m$, was given in [4]. However, it is not possible to give a constructive method for nets with $t$ uniformly small, that is, independent of $m$, and of the dimension $s$. (See [5].)

Another attempt in solving this practical problem is to provide tables of digital nets. Some intensive computer calculation was done in this direction in [1]. For example, there is given a table of $(t, m, s)$-nets to base 2 for $s=4$ and $2 \leq m \leq 20$, and for $5 \leq s \leq 12$ and $2 \leq m \leq 10$, with values of $t$ between 1 and 6 . Of course, this table, for most practical purposes, still is too small.

In this paper we have tried an alternative approach for managing the practical problems, which is described in the next section.

\section{THE METHOD AND THE RESULTS}

We only use $(0, m, s)$-nets to a base $2^{h}(h \in \mathbb{N} ; h \geq 2)$ for the integration of base- 2 Walsh series.

That is: We have to integrate a base-2 Walsh series of dimension $s \geq 4$. We choose $h$ minimal such that $2^{h} \geq s-1$. Then by Niederreiter's construction method we construct a digital $(0, m, s)$-net to base $2^{h}$ and use this net for the numerical integration of the function.

To test the quality of this procedure, we of course first have to provide a result on the integration of base- 2 functions by base- $2^{h}$ digital nets. The basis for such a result is the following Theorem 3, which gives a relation between the Walsh coefficients of a function represented in different bases.

Theorem 3. For $h, s \in \mathbb{N}, c>0$ we have: Here,

(a) If $f \in{ }_{2} \bar{E}_{s}^{\alpha}(c)$, then $f \in{ }_{2^{h}} \bar{E}_{s}^{\alpha-\beta_{h}}\left(c \cdot 2^{h s \alpha}\right)$, provided that $\alpha>1+\beta_{h}$.

$$
\beta_{h}=\frac{h-1}{2 h}+\frac{\sum_{k=0}^{h-2} \log \sin \left(\frac{\pi}{4}+\frac{\pi}{2}\left\{\frac{4^{\left[\frac{k}{2}\right]}-1}{3 \cdot 2^{k+1}}\right\}\right)}{h \cdot \log 2},
$$

and $\beta_{h}$ is monotonically increasing with

$$
\beta:=\lim _{h \rightarrow \infty} \beta_{h}=\frac{1}{2}+\frac{\log \sin \frac{5 \pi}{12}}{\log 2}=0.4499 \ldots
$$

(b) The "exponents" $\alpha-\beta_{h}$ in the above result are best possible.

Remark. We have $\beta_{2}=0.25, \beta_{3}=0.2952 \ldots, \beta_{4}=0.3394 \ldots, \beta_{5}=$ $0.3588 \ldots$. .

From Theorems 1 and 3 the following result immediately follows, and the following conjecture is suggested.

Theorem 4. If $\underline{x}_{1}, \ldots, \underline{x}_{N}$ is a $(0, m, s)$-net to base $2^{h}$, then

$$
R_{N}(f) \leq K\left(s, \alpha, c \cdot 2^{h s \alpha}\right) \cdot \frac{(\log N)^{s-1}}{N^{\alpha-\beta_{h}-1}}
$$

for all $f \in{ }_{2} \bar{E}_{s}^{\alpha}(c)$ with $\alpha>1+\beta_{h}$. 
Conjecture. If $\underline{x}_{1}, \ldots, \underline{x}_{N}$ is a digital $(0, m, s)$-net to base $2^{h}$, then

$$
R_{N}(f) \leq K\left(s, \alpha, c \cdot 2^{h s \alpha}\right) \cdot \frac{(\log N)^{s-1}}{N^{\alpha-\beta_{h}}}
$$

for all $f \in{ }_{2} \bar{E}_{s}^{\alpha}(c)$ with $\alpha>1+\beta_{h}$.

(Here, $K\left(s, \alpha, c \cdot 2^{h s \alpha}\right)$ means the same constant as in Theorem 1, with $c \cdot 2^{h s \alpha}$ replacing $c$.) So the integration error with this method is larger by a factor $N^{\beta_{h}}$ at most. But on the other hand, the method provides a concrete effective device to integrate base-2 Walsh series in arbitrary dimension with arbitrarily large $N$ and an integration error of at most $\sim(\log N)^{s-1} / N^{\alpha-\beta_{h}-1}$, and most probably of at most $\sim(\log N)^{s-1} / N^{\alpha-\beta_{h}}$, which still is a very useful order if $\alpha$ is not too small.

Concrete examples and calculations will be given in the last section of the paper.

\section{PROOF OF THE RESULTS}

We only have to prove Theorem 3 . Let $h \in \mathbb{N}$ be given and $B=2^{h}$. We write $\phi$ and wal for Rademacher, resp. Walsh functions to base 2. Since for $0 \leq j<h, \phi_{j}$ is constant on intervals of the form $\left[\frac{a}{B}, \frac{a+1}{B}\right)$, it is obvious that for $n \geq B$ and all $j$ with $0 \leq j<h$ we have

$$
\int_{0}^{1} \phi_{j}(x) \cdot \overline{\mathrm{wal}}_{n}^{(B)}(x) d x=0,
$$

and therefore

$$
\phi_{j}(x)=\sum_{n=0}^{B-1} A(j, n) \cdot \mathrm{wal}_{n}^{(B)}(x) .
$$

Here and in the following we sometimes make use of the following basic principle for Walsh series (see [3]):

(*) $\quad$ For given base $b \geq 2$ and $k, l \in \mathbb{N}_{0}$, let $k \oplus l$ denote digital summation modulo $b$ of $k$ and $l$. Then $\operatorname{wal}_{k}^{(b)}(x) \cdot \operatorname{wal}_{l}^{(b)}(x)=$ $\mathrm{wal}_{k \oplus l}^{(b)}(x)$.

Therefore, for $0 \leq k<B$,

$$
\operatorname{wal}_{k}(x)=\sum_{n=0}^{B-1} \gamma(k, n) \cdot \mathrm{wal}_{n}^{(B)}(x),
$$

and with $s \in \mathbb{N}$,

$$
\begin{aligned}
\operatorname{wal}_{k \cdot B^{s}}(x) & =\operatorname{wal}_{k}\left(B^{s} x\right)=\sum_{n=0}^{B-1} \gamma(k, n) \cdot \operatorname{wal}_{n}^{(B)}\left(B^{s} x\right) \\
& =\sum_{n=0}^{B-1} \gamma(k, n) \cdot\left(\phi_{s}^{(B)}(x)\right)^{n}=\sum_{n=0}^{B-1} \gamma(k, n) \cdot \operatorname{wal}_{n \cdot B^{s}}^{(B)}(x) .
\end{aligned}
$$

Here,

$$
\gamma(k, n)=\int_{0}^{1} \operatorname{wal}_{k}(x) \cdot\left(\phi_{0}^{(B)}(x)\right)^{-n} d x .
$$

Note that $\gamma(k, 0)=0$ whenever $k \neq 0$. 
Let $v_{k}:=\left(\operatorname{wal}_{k}(0), \ldots, \operatorname{wal}_{k}\left(\frac{B-1}{B}\right)\right), w_{n}:=\left(\left(w^{-n}\right)^{0}, \ldots,\left(w^{-n}\right)^{B-1}\right)^{T}, k=$ $0, \ldots, B-1, n=0, \ldots, B-1$, with $w=e^{2 \pi i / B}$, and

$$
V^{(h)}:=\left(\begin{array}{c}
v_{0} \\
\vdots \\
v_{B-1}
\end{array}\right), \quad W^{(h)}:=\left(w_{0}, \ldots, w_{B-1}\right) .
$$

Then $C^{(h)}:=(\gamma(k, n))_{k, n=0, \ldots, B-1}=\frac{1}{B} \cdot V^{(h)} \cdot W^{(h)}$. Let

$$
M_{h}:=\max _{n=0, \ldots, B-1} \sum_{k=0}^{B-1}|\gamma(k, n)|=: \sum_{k=0}^{B-1}\left|\gamma\left(k, n_{0}\right)\right| \quad \text { and } \quad \beta_{h}^{\prime}:=\frac{\log M_{h}}{h \cdot \log 2} .
$$

We now split the proof into several lemmas.

Lemma 1. If $g \in{ }_{2} \bar{E}_{1}^{\alpha}(c)$, then $g \in{ }_{B} \bar{E}_{1}^{\alpha-\beta_{h}^{\prime}}\left(c \cdot B^{\alpha}\right)$, provided that $\alpha>1+\beta_{h}^{\prime}$. The exponent $\alpha-\beta_{h}^{\prime}$ is best possible.

Proof. Let $n=v_{0}(n)+v_{1}(n) B+\cdots+v_{j}(n) B^{j}, v_{i}(n) \in\{0, \ldots, B-1\}$ for $i=0, \ldots, j-1, v_{j}(n) \in\{1, \ldots, B-1\}$. Then

$$
\begin{aligned}
g(x) & :=g_{0}+\sum_{j=0}^{\infty} \sum_{n=B^{j}}^{B^{j+1}-1} g_{n} \cdot \prod_{s=0}^{j} \operatorname{wal}_{v_{s}(n) \cdot B^{s}}(x) \\
& =g_{0}+\sum_{j=0}^{\infty} \sum_{n=B^{j}}^{B^{j+1}-1} g_{n} \cdot \prod_{s=0}^{j}\left(\sum_{m=0}^{B-1} \gamma\left(v_{s}(n), m\right) \cdot \mathrm{wal}_{m \cdot B^{s}}^{(B)}(x)\right) \\
& =\sum_{l=0}^{\infty} h_{l} \cdot \mathrm{wal}_{l}^{(B)}(x) .
\end{aligned}
$$

Since $v_{j}(n) \neq 0$, we have $\gamma\left(v_{j}(n), 0\right)=0$, and therefore by the principle $(*)$,

$$
\sum_{n=B^{j}}^{B^{j+1}-1} g_{n} \cdot \prod_{s=0}^{j}\left(\sum_{m=0}^{B-1} \gamma\left(v_{s}(n), m\right) \cdot \mathrm{wal}_{m \cdot B^{s}}^{(B)}(x)\right)=\sum_{l=B^{j}}^{B^{j+1}-1} h_{l} \cdot \mathrm{wal}_{l}^{(B)}(x) .
$$

Let $l=u_{j} B^{j}+\cdots+u_{0}, u_{i} \in\{0, \ldots, B-1\}$ for $i=0, \ldots, j-1, u_{j} \in$ $\{1, \ldots, B-1\}$. Then

$$
\begin{aligned}
h_{l} \cdot \mathrm{wal}_{l}^{(B)}(x) & =h_{l} \cdot \prod_{s=0}^{j} \mathrm{wal}_{u_{s} \cdot B^{s}}^{(B)}(x) \\
& =\sum_{n=B^{j}}^{B^{j+1}-1} g_{n} \cdot \prod_{s=0}^{j} \gamma\left(v_{s}(n), u_{s}\right) \cdot \mathrm{wal}_{u_{s} \cdot B^{s}}^{(B)}(x) \\
& =\left[\sum_{n=B^{j}}^{B^{j+1}-1}\left(g_{n} \cdot \prod_{s=0}^{j} \gamma\left(v_{s}(n), u_{s}\right)\right)\right] \cdot \mathrm{wal}_{l}^{(B)}(x),
\end{aligned}
$$

so that

$$
h_{l}=\sum_{n=B^{j}}^{B^{j+1}-1}\left(g_{n} \cdot \prod_{s=0}^{j} \gamma\left(v_{s}(n), u_{s}\right)\right)
$$


and

$$
\left|h_{l}\right| \leq c \cdot \sum_{n=B^{j}}^{B^{j+1}-1} \frac{1}{n^{\alpha}} \cdot \prod_{s=0}^{j}\left|\gamma\left(v_{s}(n), u_{s}\right)\right|=: c \cdot E_{l} .
$$

If we consider the function $\tilde{g} \in{ }_{2} \bar{E}_{1}^{\alpha}(c)$ with

$$
\tilde{g}_{n}=\frac{c}{n^{\alpha}} \cdot \frac{\prod_{s=0}^{j} \bar{\gamma}\left(v_{s}(n), u_{s}\right)}{\prod_{s=0}^{j}\left|\gamma\left(v_{s}(n), u_{s}\right)\right|}
$$

then we have $h_{l}=c \cdot E_{l}$. Furthermore,

$$
\frac{1}{B^{(j+1) \alpha}} \cdot \sum_{n=B^{j}}^{B^{j+1}-1} \prod_{s=0}^{j}\left|\gamma\left(v_{s}(n), u_{s}\right)\right|<E_{l} \leq \frac{1}{B^{j \alpha}} \cdot \sum_{n=B^{j}}^{B^{j+1}-1} \prod_{s=0}^{j}\left|\gamma\left(v_{s}(n), u_{s}\right)\right| .
$$

Because of $\gamma\left(0, u_{j}\right)=0$, we have

$$
\begin{aligned}
\sum_{n=B^{j}}^{B^{j+1}-1} & \prod_{s=0}^{j}\left|\gamma\left(v_{s}(n), u_{s}\right)\right|=\sum_{n=0}^{B^{j+1}-1} \prod_{s=0}^{j}\left|\gamma\left(v_{s}(n), u_{s}\right)\right| \\
& =\sum_{v_{0}=0}^{B-1} \cdots \sum_{v_{j}=0}^{B-1} \prod_{s=0}^{j}\left|\gamma\left(v_{s}, u_{s}\right)\right|=\prod_{s=0}^{j}\left(\sum_{v=0}^{B-1}\left|\gamma\left(v, u_{s}\right)\right|\right) .
\end{aligned}
$$

Therefore always

$$
E_{l} \leq \frac{1}{B^{j \alpha}} \cdot\left(M_{h}\right)^{j+1} \leq B^{\alpha} \cdot \frac{1}{l^{\alpha-\beta_{h}^{\prime}}},
$$

and, if $l=n_{0} \cdot\left(B^{j+1}-1\right) /(B-1)$,

$$
E_{l} \geq \frac{1}{B^{(j+1) \alpha}} \cdot\left(M_{h}\right)^{j+1} \geq \frac{1}{B^{\alpha-\beta_{h}^{\prime}}} \cdot \frac{1}{l^{\alpha-\beta_{h}^{\prime}}},
$$

and the result follows.

Remark. From the proof above it follows that, if we set

$$
\Delta_{h}:=\sup _{j} \max _{B^{j} \leq l<B^{j+1}} l^{\alpha-\beta_{h}^{\prime}} \cdot \sum_{n=B^{j}}^{B^{j+1}-1} \frac{1}{n^{\alpha}} \cdot \prod_{s=0}^{j}\left|\gamma\left(v_{s}(n), u_{s}(l)\right)\right|,
$$

then the result " $g \in{ }_{2} \bar{E}_{1}^{\alpha}(c) \rightarrow g \in{ }_{B} \bar{E}_{1}^{\alpha-\beta_{h}^{\prime}}\left(c \cdot \Delta_{h}\right)$ " is true and best possible, even in the constants.

Lemma 2. If $f \in{ }_{2} \bar{E}_{s}^{\alpha}(c)$, then $f \in{ }_{B} \bar{E}_{s}^{\alpha-\beta_{h}^{\prime}}\left(c \cdot B^{s \alpha}\right)$, provided that $\alpha>1+\beta_{h}^{\prime}$. The exponent $\alpha-\beta_{h}^{\prime}$ is best possible. 
Proof. That $\alpha-\beta_{h}^{\prime}$ is best possible clearly follows from Lemma 1. We only have to consider the function $\tilde{g}$ defined in the proof of Lemma 1 as a function which is constant in $s-1$ variables.

Now we use induction on $s$. The case $s=1$ is settled by Lemma 1. Assuming the assertion true for dimension $\leq s-1$, we get: Let $f \in{ }_{2} \bar{E}_{s}^{\alpha}(c), s \geq 2$, and $\alpha>1+\beta_{h}^{\prime}$ with

$$
\begin{aligned}
& f\left(x_{1}, \ldots, x_{s}\right):=\sum_{k_{1}, \ldots, k_{s}=0}^{\infty} \hat{f}\left(k_{1}, \ldots, k_{s}\right) \cdot \operatorname{wal}_{k_{1}}\left(x_{1}\right) \cdots \operatorname{wal}_{k_{s}}\left(x_{s}\right) \\
& \quad=: \sum_{k_{1}=0}^{\infty}\left(\sum_{k_{2}, \ldots, k_{s}=0}^{\infty} \hat{f}^{\left(k_{1}\right)}\left(k_{2}, \ldots, k_{s}\right) \cdot \operatorname{wal}_{k_{2}}\left(x_{2}\right) \cdots \operatorname{wal}_{k_{s}}\left(x_{s}\right)\right) \cdot \operatorname{wal}_{k_{1}}\left(x_{1}\right) .
\end{aligned}
$$

Then

$$
\left|\hat{f}^{\left(k_{1}\right)}\left(k_{2}, \ldots, k_{s}\right)\right| \leq \frac{c}{\bar{k}_{1}^{\alpha}} \cdot \frac{1}{\left(\bar{k}_{2} \ldots \bar{k}_{s}\right)^{\alpha}},
$$

and by the induction hypothesis we have that the inner sum above is equal to

$$
\sum_{j_{2}, \ldots, j_{s}=0}^{\infty} h^{\left(k_{1}\right)}\left(j_{2}, \ldots, j_{s}\right) \cdot \mathbf{w a l}_{j_{2}}^{(\boldsymbol{B})}\left(x_{2}\right) \cdots \mathrm{wal}_{j_{s}}^{(\boldsymbol{B})}\left(x_{s}\right)
$$

with

$$
\left|h^{\left(k_{1}\right)}\left(j_{2}, \ldots, j_{s}\right)\right| \leq B^{(s-1) \cdot \alpha} \cdot \frac{c}{\bar{k}_{1}^{\alpha}} \cdot \frac{1}{\left(\bar{j}_{2} \cdots \bar{j}_{s}\right)^{\alpha-\beta_{h}^{\prime}}} .
$$

Therefore (rearranging of the summands provides no problem since $\alpha>1+\beta_{h}^{\prime}$ ),

$$
\begin{aligned}
f\left(x_{1}, \ldots, x_{s}\right)= & \sum_{j_{2}, \ldots, j_{s}=0}^{\infty} \operatorname{wal}_{j_{2}}^{(B)}\left(x_{2}\right) \cdots \mathrm{wal}_{j_{s}}^{(B)}\left(x_{s}\right) \\
& \cdot \sum_{k_{1}=0}^{\infty} h^{\left(k_{1}\right)}\left(j_{2}, \ldots, j_{s}\right) \cdot \operatorname{wal}_{k_{1}}\left(x_{1}\right) .
\end{aligned}
$$

Again, using the induction hypothesis for the last sum, we get the result.

Lemma 3. For $j$ with $0 \leq j<B, j=j_{h-1} 2^{h-1}+\cdots+j_{0}$, let $J_{k}:=$ $\left(j_{k} 2^{k}+\cdots+j_{0}\right) / 2^{k+1}$. Then

$$
M_{h}=(\sqrt{2})^{h-1} \cdot \max _{0 \leq j<B} \cdot \prod_{k=0}^{h-2} \sin \left(\frac{\pi}{4}+\frac{\pi}{2} J_{k}\right) .
$$

Proof. Let $i=e_{h-1} 2^{h-1}+\cdots+e_{0}$ and $v_{i}:=\left(v_{i, 0}, \ldots, v_{i, B-1}\right)$ be the $i$ th row of $V^{(h)}$. By the definition of $V^{(h)}$ and by the definition of Walsh functions, we have $\left(v_{i, 0}, \ldots, v_{i, 2^{k-1}-1}\right)=\left(v_{i, 2^{k-1}}, \ldots, v_{i, 2^{k}-1}\right)$ if and only if $e_{h-k}=$ 0 . If $e_{h-k}=1$, then $\left(v_{i, 0}, \ldots, v_{i, 2^{k-1}-1}\right)=-\left(v_{i, 2^{k-1}}, \ldots, v_{i, 2^{k}-1}\right)$. (This follows since $\operatorname{wal}_{i}\left(2^{k-1} / 2^{h}\right)=(-1)^{e_{h-k}}$ and $\operatorname{wal}_{i}(0)=1$.) Therefore, with 


$$
\begin{aligned}
& \varepsilon_{k}:=1-2 e_{k} \text {, we get } \\
& 2^{h} \cdot|\gamma(i, n)|=\left|v_{i} \cdot w_{n}\right| \\
& =\mid\left(v_{i, 0}, \ldots, v_{i, 2^{h-1}-1}\right) \cdot\left(\begin{array}{c}
\left(w^{-n}\right)^{0} \\
\vdots \\
\left(w^{-n}\right)^{2^{h-1}-1}
\end{array}\right) \\
& +\varepsilon_{0} \cdot\left(v_{i, 0}, \ldots, v_{i, 2^{h-1}-1}\right) \cdot\left(w^{-n}\right)^{2^{h-1}} \cdot\left(\begin{array}{c}
\left(w^{-n}\right)^{0} \\
\vdots \\
\left(w^{-n}\right)^{2^{h-1}-1}
\end{array}\right) \\
& =\left|\left(v_{i, 0}, \ldots, v_{i, 2^{h-1}-1}\right) \cdot\left(\begin{array}{c}
\left(w^{-n}\right)^{0} \\
\vdots \\
\left(w^{-n}\right)^{2^{h-1}-1}
\end{array}\right) \cdot\left(1+\left(w^{-n}\right)^{2^{h-1}} \cdot \varepsilon_{0}\right)\right| \\
& =\cdots=\prod_{k=0}^{h-1}\left|1+\left(w^{-n}\right)^{2^{k}} \cdot \varepsilon_{h-1-k}\right| \\
& =2^{h} \cdot \prod_{k=0}^{h-1}\left|\operatorname{Trig}_{k}\left(2 \pi \frac{-n}{2^{h-k+1}}\right)\right| \text {, }
\end{aligned}
$$

where

$$
\operatorname{Trig}_{k}= \begin{cases}\cos & \text { if } \varepsilon_{h-1-k}=1 \\ \sin & \text { if } \varepsilon_{h-1-k}=-1\end{cases}
$$

Hence,

$$
\sum_{i=0}^{2^{h}-1}|\gamma(i, n)|=\prod_{k=0}^{h-1}\left(\left|\cos \left(2 \pi \frac{n}{2^{h-k+1}}\right)\right|+\left|\sin \left(2 \pi \frac{n}{2^{h-k+1}}\right)\right|\right) .
$$

Now $t(x):=|\cos (x)|+|\sin (x)|$ is periodic with period $\frac{\pi}{2}$ and $t(x)=$ $\sqrt{2} \sin \left(\frac{\pi}{4}+x\right)$ for $0 \leq x \leq \frac{\pi}{2}$, so that the last expression is equal to

$$
\sqrt{2}^{h-1} \cdot \prod_{k=0}^{h-2} \sin \left(\frac{\pi}{4}+\frac{\pi}{2}\left\{\frac{n}{2^{h-k-1}}\right\}\right)
$$

and the assertion of Lemma 3 immediately follows.

Lemma 4. For $j=j_{h-1} 2^{h-1}+\cdots+j_{0}$, let $J_{k}:=\left(j_{k} 2^{k}+\cdots+j_{0}\right) / 2^{k+1}$ and

$$
F_{h}(j):=\prod_{k=0}^{h-2} \sin \left(\frac{\pi}{4}+\frac{\pi}{2} J_{k}\right) \text {. }
$$

Then

$$
\max _{0 \leq j<B} F_{h}(j)=F_{h}(T(h))
$$

where

$$
T(h)= \begin{cases}2^{h-3}+2^{h-5}+\cdots+2^{0} & \text { for } h \text { odd }, \\ 2^{h-2}+2^{h-4}+\cdots+2^{0} & \text { for } h \text { even. }\end{cases}
$$

Proof. This is easily checked for $h=1,2$. For $h \geq 3$ we have $F_{h}(0)=$ $1 / \sqrt{2}^{h-1}$ and $F_{h}\left(2^{h-2}\right)=1 / \sqrt{2}^{h-2}$. These certainly are not the only maxima 
for $h \geq 3$ since, for example, $F_{h}\left(2^{h-2}+2^{h-3}\right)$ obviously gives at least as large a value. Further, it is easily checked that $F_{h}(k)=F_{h}\left(2^{h-1}-k\right)$ for $k=1, \ldots, 2^{h-2}-1$. We proceed by induction on $h$. Let the assertion be true for $F_{k}$ with $1 \leq k \leq h-1$. If $h$ is odd, we consider $j$ with $0<j<2^{h-2}$ arbitrary but $j \neq T(h)$. Let $T(h):=t_{h-3} t_{h-2} \cdots t_{0}$ and $j:=j_{h-3} j_{h-2} \cdots j_{0}$ be the representations to base 2 , and let $l$ be the maximal index such that $j_{l} \neq t_{l}$.

Case 1: $l$ even. Then $t_{l}=1$ and $j_{l}=0$. Further,

$$
\begin{gathered}
F_{h}(j)=\prod_{k=0}^{l} \sin \left(\frac{\pi}{2}\left(\frac{1}{2}+J_{k}\right)\right) \cdot \prod_{k=l+1}^{h-2} \sin \left(\frac{\pi}{2}\left(\frac{1}{2}+J_{k}\right)\right)=: \Pi_{1} \cdot \Pi_{2}, \\
F_{h}(T(h))=\prod_{k=0}^{l} \sin \left(\frac{\pi}{2}\left(\frac{1}{2}+T(h)_{k}\right)\right) \cdot \prod_{k=l+1}^{h-2} \sin \left(\frac{\pi}{2}\left(\frac{1}{2}+T(h)_{k}\right)\right)=: \Pi_{1}^{\prime} \cdot \Pi_{2}^{\prime} .
\end{gathered}
$$

We have

$$
\begin{aligned}
& \Pi_{1}^{\prime}=F_{l+2}(T(l+2)), \quad l+2<h, \\
& \Pi_{1}=F_{l+2}\left(j_{l+1} \cdots j_{0}\right),
\end{aligned}
$$

so that by the induction hypothesis, $\Pi_{1} \leq \Pi_{1}^{\prime}$. It remains to show that $\Pi_{2} \leq \Pi_{2}^{\prime}$.

Let $\tau:=t_{l} \cdots t_{0}-j_{l} \cdots j_{0}$; then $0 \leq \tau \leq 2^{l}+2^{l-2}+\cdots+2^{0}$, and for $k \geq l+1$ we have

$$
\sin \left(\frac{\pi}{2}\left(\frac{1}{2}+J_{k}\right)\right)=\sin \left(\frac{\pi}{2}\left(\frac{1}{2}+T(h)_{k}-\frac{\tau}{2^{k+1}}\right)\right) .
$$

Hence,

$$
\begin{aligned}
\Pi_{2}= & \sin \left(\frac{\pi}{2}\left(\frac{1}{2}+T(h)_{h-2}-\frac{\tau}{2^{h-1}}\right)\right) \\
& \cdot \prod_{k=(l+2) / 2}^{(h-3) / 2} \sin \left(\frac{\pi}{2}\left(\frac{1}{2}+T(h)_{2 k}-\frac{\tau}{2^{2 k+1}}\right)\right) \\
& \cdot \sin \left(\frac{\pi}{2}\left(\frac{1}{2}+T(h)_{2 k-1}-\frac{\tau}{2^{2 k}}\right)\right)
\end{aligned}
$$

and

$$
\begin{aligned}
\Pi_{2}^{\prime}= & \sin \left(\frac{\pi}{2}\left(\frac{1}{2}+T(h)_{h-2}\right)\right) \\
& \cdot \prod_{k=(l+2) / 2}^{(h-3) / 2} \sin \left(\frac{\pi}{2}\left(\frac{1}{2}+T(h)_{2 k}\right)\right) \cdot \sin \left(\frac{\pi}{2}\left(\frac{1}{2}+T(h)_{2 k-1}\right)\right) .
\end{aligned}
$$

Because of $0 \leq T(h)_{h-2}-\tau / 2^{h-1} \leq T(h)_{h-2}<\frac{1}{2}$, we have

$$
\sin \left(\frac{\pi}{2}\left(\frac{1}{2}+T(h)_{h-2}-\frac{\tau}{2^{h-1}}\right)\right) \leq \sin \left(\frac{\pi}{2}\left(\frac{1}{2}+T(h)_{h-2}\right)\right),
$$

so that it suffices to show that for all $k=\frac{l+2}{2}, \ldots, \frac{h-3}{2}$ we have

$$
\begin{aligned}
& \sin \left(\frac{\pi}{2}\left(\frac{1}{2}+T(h)_{2 k}-\frac{\tau}{2^{2 k+1}}\right)\right) \cdot \sin \left(\frac{\pi}{2}\left(\frac{1}{2}+T(h)_{2 k-1}-\frac{\tau}{2^{2 k}}\right)\right) \\
& \quad \leq \sin \left(\frac{\pi}{2}\left(\frac{1}{2}+T(h)_{2 k}\right)\right) \cdot \sin \left(\frac{\pi}{2}\left(\frac{1}{2}+T(h)_{2 k-1}\right)\right) .
\end{aligned}
$$


We have $T(h)_{2 k}=\frac{1}{2}+T(h)_{2 k-1} / 2$. Let

$$
\varphi:=\varphi_{k}:=\frac{\pi}{4}\left(T(h)_{2 k-1}-\frac{\tau}{2^{2 k}}\right), \quad \sigma:=\sigma_{k}:=\frac{\pi}{4} T(h)_{2 k-1} ;
$$

then we have to show

$$
\sin \left(\frac{\pi}{2}+\varphi\right) \cdot \sin \left(\frac{\pi}{4}+2 \varphi\right) \leq \sin \left(\frac{\pi}{2}+\sigma\right) \cdot \sin \left(\frac{\pi}{4}+2 \sigma\right) .
$$

Here, $0 \leq \varphi \leq \sigma \leq \frac{\pi}{12}$. Since $f(x):=\sin \left(\frac{\pi}{2}+x\right) \cdot \sin \left(\frac{\pi}{4}+2 x\right)$ is monotonically increasing on $\left[0, \frac{\pi}{12}\right]$, the result in Case 1 follows.

Case 2: $l$ odd. Then $t_{l}=0, j_{l}=1$ (and $\left.l \leq h-4\right)$. By splitting up $F_{h}(j)$ and $F_{h}(T(h))$ in the same way as above, we again have

$$
\Pi_{1}^{\prime}=F_{l+2}(T(l+2)) \geq \Pi_{1},
$$

and it again remains to show that $\Pi_{2} \leq \Pi_{2}^{\prime}$.

Now let $\tau:=j_{l} \cdots j_{0}-t_{l} \cdots t_{0}$; then $0 \leq \tau \leq 2^{l}+2^{l-2}+\cdots+2^{0}$, and for $k \geq l+1$ we have

$$
\sin \left(\frac{\pi}{2}\left(\frac{1}{2}+J_{k}\right)\right)=\sin \left(\frac{\pi}{2}\left(\frac{1}{2}+T(h)_{k}+\frac{\tau}{2^{k+1}}\right)\right),
$$

and hence

$$
\begin{aligned}
\Pi_{2}= & \prod_{k=(l+1) / 2}^{(h-3) / 2} \sin \left(\frac{\pi}{2}\left(\frac{1}{2}+T(h)_{2 k+1}+\frac{\tau}{2^{2 k+2}}\right)\right) \\
& \cdot \sin \left(\frac{\pi}{2}\left(\frac{1}{2}+T(h)_{2 k}+\frac{\tau}{2^{2 k+1}}\right)\right)
\end{aligned}
$$

and

$$
\Pi_{2}^{\prime}=\prod_{k=(l+1) / 2}^{(h-3) / 2} \sin \left(\frac{\pi}{2}\left(\frac{1}{2}+T(h)_{2 k+1}\right)\right) \cdot \sin \left(\frac{\pi}{2}\left(\frac{1}{2}+T(h)_{2 k}\right)\right),
$$

so that it suffices to show that for all $k=\frac{l+1}{2}, \ldots, \frac{h-3}{2}$ we have

$$
\begin{aligned}
& \sin \left(\frac{\pi}{2}\left(\frac{1}{2}+T(h)_{2 k+1}+\frac{\tau}{2^{2 k+2}}\right)\right) \cdot \sin \left(\frac{\pi}{2}\left(\frac{1}{2}+T(h)_{2 k}+\frac{\tau}{2^{2 k+1}}\right)\right) \\
& \quad \leq \sin \left(\frac{\pi}{2}\left(\frac{1}{2}+T(h)_{2 k+1}\right)\right) \cdot \sin \left(\frac{\pi}{2}\left(\frac{1}{2}+T(h)_{2 k}\right)\right) .
\end{aligned}
$$

We have $T(h)_{2 k+1}=T(h)_{2 k} / 2$. Now let $\varphi:=\varphi_{k}:=\frac{\pi}{4}\left(T(h)_{2 k}+\tau / 2^{2 k+1}\right)$ and $\sigma:=\sigma_{k}:=\frac{\pi}{4} T(h)_{2 k} ;$ then we have to show

$$
\sin \left(\frac{\pi}{4}+\varphi\right) \cdot \sin \left(\frac{\pi}{4}+2 \varphi\right) \leq \sin \left(\frac{\pi}{4}+\sigma\right) \cdot \sin \left(\frac{\pi}{4}+2 \sigma\right) .
$$

Here, $\frac{5 \pi}{32} \leq \sigma \leq \varphi \leq \frac{\pi}{4}$. Since $f(x):=\sin \left(\frac{\pi}{4}+x\right) \cdot \sin \left(\frac{\pi}{4}+2 x\right)$ is monotonically decreasing in $\left[\frac{5 \pi}{32}, \frac{\pi}{4}\right]$, the result for $h$ odd follows. 
If $h$ is even, then we consider $j$ with $2^{h-2}<j<2^{h-1}, j \neq T(h)$. If we define $l$ as above, we again have $l \leq h-3$, and we can argue quite analogously to the previous case. The result follows.

Finishing the proof of Theorem 3. We now have that $\beta_{h}^{\prime}$ from Lemma 2 is

$$
\begin{gathered}
\beta_{h}^{\prime}=\frac{\log M_{h}}{h \cdot \log 2}=\frac{h-1}{2 h}+\frac{\sum_{k=0}^{h-2} \log \sin \left(\frac{\pi}{4}+\frac{\pi}{2} \cdot T(h)_{k}\right)}{h \cdot \log 2}, \\
T(h)= \begin{cases}\frac{4^{(h-1) / 2}-1}{3} & \text { if } h \text { odd }, \\
\frac{4^{h / 2}-1}{3} & \text { if } h \text { even, }\end{cases}
\end{gathered}
$$

and $T(h)_{k}=\left\{T(h) / 2^{k+1}\right\}$, so that

$$
\begin{array}{ll}
\beta_{h}^{\prime}=\frac{h-1}{2 h}+\frac{\sum_{k=0}^{h-2} \log \sin \left(\frac{\pi}{4}+\frac{\pi}{2}\left\{\frac{4^{(h-1) / 2}-1}{3 \cdot 2^{k+1}}\right\}\right)}{h \cdot \log 2} & \text { if } h \text { odd, } \\
\beta_{h}^{\prime}=\frac{h-1}{2 h}+\frac{\sum_{k=0}^{h-2} \log \sin \left(\frac{\pi}{4}+\frac{\pi}{2}\left\{\frac{4^{h / 2}-1}{3 \cdot 2^{k+1}}\right\}\right)}{h \cdot \log 2} & \text { if } h \text { even, }
\end{array}
$$

and therefore $\beta_{h}^{\prime}=\beta_{h}$.

That $\beta_{h}$ is increasing follows directly from the fact that by construction $C^{(h)}$ is always a submatrix of $C^{(h+1)}$ and from the definition of $\beta_{h}^{\prime}$. Since $k \leq h-2$, and therefore $k+1 \leq 2 \cdot\left[\frac{h}{2}\right]$, and since $\left\{\frac{1}{3} \cdot 2^{m}\right\}=\frac{1}{3} \cdot 2^{m \bmod 2}$, we have

$$
\left\{\frac{4^{[h / 2]}}{3 \cdot 2^{k+1}}\right\}= \begin{cases}\frac{2}{3} & \text { for } k \text { even } \\ \frac{1}{3} & \text { for } k \text { odd. }\end{cases}
$$

Therefore, because of $0<1 /\left(3 \cdot 2^{k+1}\right)<1 / 3$,

$$
\left\{\frac{4^{[h / 2]}-1}{3 \cdot 2^{k+1}}\right\}= \begin{cases}\frac{2}{3}-\frac{1}{3 \cdot 2^{k+1}} & \text { for } k \text { even }, \\ \frac{1}{3}-\frac{1}{3 \cdot 2^{k+1}} & \text { for } k \text { odd },\end{cases}
$$

and because of $\sin \left(\frac{5 \pi}{12}+\frac{\pi}{2} x\right)=\sin \left(\frac{7 \pi}{12}-\frac{\pi}{2} x\right)$, we have

$$
\sin \left(\frac{\pi}{4}+\frac{\pi}{2} \cdot\left\{\frac{4^{[h / 2]}-1}{3 \cdot 2^{k+1}}\right\}\right)=\sin \left(\frac{5 \pi}{12}+\frac{\pi}{2} \cdot \frac{(-1)^{k}}{3 \cdot 2^{k+1}}\right) .
$$

From this, the fact that

$$
\lim _{h \rightarrow \infty} \beta_{h}=\frac{1}{2}+\frac{\log \sin \frac{5 \pi}{12}}{\log 2}
$$

quickly follows. This finishes the proof of Theorem 3 and consequently of Theorem 4.

\section{CONCRETE EXAMPLES AND CAlCUlations}

For the construction of digital $(0, m, s)$-nets to base $2^{h}$ we use the construction method of Niederreiter, cited in $\S 1$, which was developed in [6].

As testing functions we use the following class of functions. (These functions have several advantages compared with the testing functions used in [3] and [10]. 
For example, variables cannot be separated, and almost all Walsh coefficients $\widehat{W}\left(k_{1}, \ldots, k_{s}\right)$ of these functions $f$ are of order $\left(\bar{k}_{1} \cdots \bar{k}_{s}\right)^{-\alpha}$ if $f \in{ }_{2} \bar{E}_{s}^{\alpha}$. Further, their variation is not too small.)

For the construction of the testing function, let $g_{\beta}:[0,1) \rightarrow \mathbb{R}$ with

$$
g_{\beta}:= \begin{cases}0 & \text { if } x=0 \\ 1 / 2^{(k-1) \beta} & \text { if } 1 / 2^{k} \leq x<1 / 2^{k-1}, k=1,2, \ldots\end{cases}
$$

( $\beta>\frac{\log 3}{\log 2}-1$ arbitrary). Then it is easy to check (we refrain from going into details regarding the properties of the testing function) that

$$
g_{\beta}=\frac{2^{\beta}}{2^{\beta+1}-1}-2^{\beta} \cdot \frac{2^{\beta+1}-2}{2^{\beta+1}-1} \cdot \sum_{j=0}^{\infty} \sum_{k=2^{j}}^{2^{j+1}-1} \frac{1}{2^{(j+1)(\beta+1)}} \cdot \mathrm{wal}_{k}^{(2)}(x),
$$

and therefore for $G_{\beta}\left(x_{1}, \ldots, x_{s}\right):=g_{\beta}\left(x_{1}\right) \cdots g_{\beta}\left(x_{s}\right)$ we have

$$
G_{\beta} \in{ }_{2} \bar{E}_{s}^{\beta+1}\left(c_{1}(\beta)\right) \text { with } c_{1}(\beta):=\left(2^{\beta} \cdot \frac{2^{\beta+1}-2}{2^{\beta+1}-1}\right)^{s} .
$$

In analogy to the above, for $x=0 . x_{1} x_{2} \ldots$ and $y=0 . y_{1} y_{2} \ldots$ represented to base 2 , we define $x \oplus y:=0 . x_{1}+y_{1} x_{2}+y_{2} \ldots$, where summation of the digits is modulo two. Then by the product rule for Walsh functions (see [3]): $\mathrm{wal}_{k}^{(2)}\left(x_{1} \oplus x_{2}\right)=\mathrm{wal}_{k}^{(2)}\left(x_{1}\right) \cdot \mathrm{wal}_{k}^{(2)}\left(x_{2}\right)$, and from the representation of $g_{\beta}$, we get

$$
h_{\beta}^{\gamma}\left(x_{1}, \ldots, x_{s}\right):=\left(g_{\beta}\left(x_{1} \oplus \cdots \oplus x_{s}\right)\right)^{\gamma} \in{ }_{2} \bar{E}_{s}^{(\beta \gamma+1) / s}\left(c_{2}(\beta, \gamma)\right)
$$

with $c_{2}(\beta, \gamma)=2^{\beta \gamma} \cdot\left(2^{\beta \gamma+1}-2\right) /\left(2^{\beta \gamma+1}-1\right)$. Moreover, the Walsh coefficients $h_{k_{1}, \ldots, k_{s}}$ of $h_{\beta}^{\gamma}$ are different from zero only if $k_{1}=\cdots=k_{s}$. If we take $f_{\beta}:[0,1)^{s} \rightarrow \mathbb{R}$ to be

$$
f_{\beta}\left(x_{1}, \ldots, x_{s}\right):=G_{\beta}\left(x_{1}, \ldots, x_{s}\right)-h_{\beta}^{s+(s-1) / \beta}\left(x_{1}, \ldots, x_{s}\right),
$$

then $f_{\beta} \in{ }_{2} \bar{E}_{s}^{\beta+1}(c)$ with $c=c_{1}(\beta)+c_{2}\left(\beta, s+\frac{s-1}{\beta}\right)$. We use these $f_{\beta}$ as testing functions. We have

$$
\int_{[0,1)^{s}} f_{\beta}(\underline{x}) d \underline{x}=\left(\frac{2^{\beta}}{2^{\beta+1}-1}\right)^{s}-\left(\frac{2^{\beta s+s-1}}{2^{\beta s+s}-1}\right) .
$$

In Table 1 we show the integration error of our testing function fer $\beta=2.0$ and for $\beta=3.0$ for bases 4 and 8 and dimensions 3 and 7 .

The exact values of the integral are:

$$
\begin{array}{ll}
\text { for } \beta=2.0 \text { and } s=3: & -0.3143895522984145 \ldots, \\
\text { for } \beta=2.0 \text { and } s=7: & -0.4801057095355622 \ldots, \\
\text { for } \beta=3.0 \text { and } s=3: & -0.3484183964183964 \ldots, \\
\text { for } \beta=3.0 \text { and } s=7: & -0.4877258612133539 \ldots
\end{array}
$$

The calculations were carried out on a Silicon Graphics Iris INDIGO with a MIPS R3000A RISC-Processor. 
TABLE 1. Concrete computer calculations

\begin{tabular}{|c|c|c|c|c|c|}
\hline$\beta$ & $b$ & $s$ & $N$ & numerical integration & integration error \\
\hline 2.0 & 4 & 3 & $\overline{16}$ & 0.1173629611730576 & $4.3175251347147203 \mathrm{E}-01$ \\
\hline 2.0 & 4 & 3 & 64 & -0.3178653717332622 & 3.4758194348477600 E-03 \\
\hline 2.0 & 4 & 3 & 256 & -0.3145006634294988 & 1.1111113108430715 E-04 \\
\hline 2.0 & 4 & 3 & 1024 & -0.3143905664620092 & 1.0141635947702454 E-06 \\
\hline 2.0 & 4 & 3 & 4096 & -0.3143896212968685 & 6.8998454061386383 E-08 \\
\hline 2.0 & 4 & 3 & 16384 & -0.3143895538144221 & 1.5160076527465094 E-09 \\
\hline 2.0 & 4 & 3 & 65536 & -0.3143895523228047 & 2.4390267583385139 E-11 \\
\hline 2.0 & 4 & 3 & 262144 & -0.3143895522990524 & 6.3787863879838369 E-13 \\
\hline 2.0 & 4 & 3 & 1048576 & -0.3143895522984270 & $1.2490009027033011 \mathrm{E}-14$ \\
\hline 2.0 & 4 & 3 & 4194304 & -0.3143895522984146 & 1.6653345369377348 E-16 \\
\hline 2.0 & 4 & 3 & 16777216 & -0.3143895522984145 & $0.0000000000000000 \mathrm{E}+00$ \\
\hline 2.0 & 8 & 7 & 64 & 0.0020300596961533 & $4.8213576923171553 \mathrm{E}-01$ \\
\hline 2.0 & 8 & 7 & 512 & -0.4612248946569917 & 1.8880814878570518 E-02 \\
\hline 2.0 & 8 & 7 & 4096 & -0.4808358634761971 & 7.3015394063491446 E-04 \\
\hline 2.0 & 8 & 7 & 32768 & -0.4801073128868772 & 1.6033513149937839 E-06 \\
\hline 2.0 & 8 & 7 & 262144 & -0.4801057174805712 & 7.9450089840449323 E-09 \\
\hline 2.0 & 8 & 7 & 2097152 & -0.4801057095962357 & 6.0673466251159880 E-11 \\
\hline 2.0 & 8 & 7 & 16777216 & -0.4801057095421429 & $6.5806804450119216 \mathrm{E}-12$ \\
\hline 3.0 & 4 & 3 & 16 & 0.0527399778075051 & 4.0115837422590150 E-01 \\
\hline 3.0 & 4 & 3 & 64 & -0.3495940715074610 & 1.1756750890645762 E-03 \\
\hline 3.0 & 4 & 3 & 256 & -0.348427623226 & 9.2268082019231201 E-06 \\
\hline 3.0 & 4 & 3 & 1024 & -0.3484184268146535 & 3.0396257066911403 E-08 \\
\hline 3.0 & 4 & 3 & 4096 & -0.3484183967716397 & 3.5324321245866486 E-10 \\
\hline 3.0 & 4 & 3 & 16384 & -0.3484183964203300 & 1.9335089085359414 E-12 \\
\hline 3.0 & 4 & 3 & 65536 & -0.3484183964184048 & 8.3266726846886741 E-15 \\
\hline 3.0 & 4 & 3 & 262144 & -0.3484183964183965 & 5.5511151231257827 E-17 \\
\hline 3.0 & 4 & 3 & 1048576 & -0.3484183964183964 & $0.0000000000000000 \mathrm{E}+00$ \\
\hline 3.0 & 4 & 3 & 4194304 & -0.3484183964183964 & $0.0000000000000000 \mathrm{E}+00$ \\
\hline 3.0 & 4 & 3 & 16777216 & -0.3484183964183964 & $0.0000000000000000 \mathrm{E}+00$ \\
\hline 3.0 & 8 & 7 & 64 & 0.0001804840649129 & $4.8790634527826676 \mathrm{E}-01$ \\
\hline 3.0 & 8 & 7 & 512 & -0.4661444031680542 & 2.1581458045299706 E-02 \\
\hline 3.0 & 8 & 7 & 4096 & -0.4881680796822815 & $4.4221846892761318 \mathrm{E}-04$ \\
\hline 3.0 & 8 & 7 & 32768 & -0.4877259237250645 & 6.2511710585688718 E-08 \\
\hline 3.0 & 8 & 7 & 262144 & -0.4877258614504577 & $2.3710378105334939 \mathrm{E}-10$ \\
\hline 3.0 & 8 & 7 & 2097152 & -0.4877258612135547 & $2.0078383400345956 \mathrm{E}-13$ \\
\hline 3.0 & 8 & 7 & 16777216 & -0.4877258612133638 & $9.9364960703951510 \mathrm{E}-15$ \\
\hline
\end{tabular}

\section{ACKNOWLEDGMENT}

The authors thank the referee whose various hints, remarks, and corrections helped to improve significantly the style and accuracy of this paper. 


\section{BIBLIOGRAPHY}

1. T. Hansen, G. L. Mullen, and H. Niederreiter, Good parameters for a class of node sets in quasi-Monte Carlo integration, Math. Comp. 61 (1993), 225-234.

2. G. Larcher, A class of low-discrepancy point-sets and its application to numerical integration by number-theoretical methods, Österreichisch-Ungarisch-Slowakisches Kolloquium über Zahlentheorie (F. Halter-Koch and R. Tichy, eds.), Grazer Math. Ber., vol. 318, Karl-Franzens-Univ. Graz, Graz, 1993, pp. 69-80.

3. G. Larcher and C. Traunfellner, On the numerical integration of Walsh series by number-theoretic methods, Math. Comp. 63 (1994), 277-291.

4. H. Niederreiter, Low-discrepancy and low-dispersion sequences, J. Number Theory 30 (1988), 51-70.

5. __ Orthogonal arrays and other combinatorial aspects in the theory of uniform point distributions in unit cubes, Discrete Math. 106/107 (1992), 361-367.

6. $\ldots$, Point sets and sequences with small discrepancy, Monatsh. Math. 104 (1987), 273337.

7. W. Ch. Schmid, Zur numerischen Integration von Walshreihen, Diplomarbeit, Universität Salzburg, 1993.

8. I. M. Sobol, The distribution of points in a cube and the approximate evaluation of integrals, Zh. Vychisl. Mat. i Mat. Fiz. 7 (1967), 784-802. (Russian)

9. __ Multidimensional quadrature formulas and Haar functions, "Nauka”, Moscow, 1969. (Russian)

10. C. Traunfellner, Zahlentheoretische Methoden zur numerischen Integration von Walshreihen, Diplomarbeit, Universität Salzburg, 1991.

(Larcher and Wolf) Institut für Mathematik, Universität Salzburg, HellbrunNerSTrasse 34, A-5020 SAlzbURG, Austria

(Schmid) Research Institute for Software Technology, Universität Salzburg, HellBRUNNERSTRASSE 34, A-5020 SAlZBURG, AUSTRIA

E-mail address: schmidw@dsb835.edvz.sbg.ac .at 